\title{
Contents, Vol. 161, 1970
}

\section{Index}

Atar, Z.: vide Kalberer, M.

Babel, J. et Stangos, N.: Lesions oculaires iatrogènes; Гaction d'un nouveau medicament contre $\Gamma$ angine de poitrine 115

Babel, J.; Gauthier, G.; Psilas, K.; Ricci, A.; Soriano, H. et Tsacopoulos, M.: Les signes oculaires dans les affections carotidiennes . . 324 Bangerter. A.: Zur Kataraktoperation nach fistulierender Operation . . 304 Bechrakis,E.: Unte $\Gamma$ suchungen des intraokularen Druckes mit de $\pi$ iAppIanationstonometer nach Goldmann 353 Benz, G.: vide Huber, P.

Bianchi, G.: vide Stucchi, C. A.

Bider, E.: 'Visual evoked response' im optischen Cortex bei Makuladegeneration 187

Bider, E.: vide Kalberer, M.

Bouzas, A.; Kokkinakis, K.; Papadakis, G. et Daikos, G.: La toxicité oculaire de Гéthambutol 361

Brack, B.: vide Deller, M.

Breitenmoser, R. und Würth, A.: Neue photographische Methode der

Schielwinkelmessung 346

Bruckner, R.: Erste persönliche Erfahrungen mit dem Vaccin antiherpé-

tique «Diamani» 104

Cambie, E.: vide Holland, Monte G.

Collignon, J. et Prijot, E.: Effet d'un collyre associant 1'acéclidine et

Tadrénaline sur la pression oculaire de patients atteints de glau-

come à angel ouvert 394

Comberg, D.: Die Ausdehnung des Akkommodationsphosphens im Gesichts-

feld 499

Comberg, D. und Lechnowitsch, Ch.: Die Verwendung des Lichtstreifen-

testes bei Vorliegen dichter Medientrübungen 504

Daicker, B.: Gewebliche Diskontinuitätszonen in der gesunden und patho-

logisch veränderten menschlichen Descemet'schen Membran . . 166 Daikos, G.: vide Bouzas, A.

Della Casa, F.: Zur Therapie des malignen Exophthalmus 145

Deller, M. et Brack, B.: Le traitement chirurgical de choix des ésotropies

obliques en V 340

Dieterich, C. E.: Die Ultrastruktur der retinalen Horizontalzellen des Men-

schen 152

Dietze, H.: vide Matthäus, W.

Ditzen, K.; Grützner, P.; Immich, H. und Mattheus, S.: Über das gün-

stigste Operationsalter bei Strabismus convergens und sursoadduc- 
torius. Beidseitige Rücklagerung des M. rectus int. bzw. Myektomie

des M. obi. inf 466

Dobrivojevic, D.: vide Rintelen, F.

VI

Index

Dheifus, M.: Klinische Erfahrungen mit hydrophilen Kontaktlinsen bei

einseitig aphaken Kindern 279

Eisner, G.: Kryoextraktion der Linse ohne Assitenz 300

Erez, Ruth: Glaucoma juvenile beim Turner-Syndrom 264

Favre, M.: Le traitement de la rétinopathie diabétique et de Thémorragie

récidivante du vitré par le Dobésilate de calcium 389

Fehér, J.: Polarization Optical Studies on the Ultrastructure of the Photo-receptor 402

Frisén, $\mathrm{L} \cdot .:$ The Cartographic Deformations of the Visual Field .... 38

Gailloud, C: vide Rosselet, E.

Gauthier, G.: vide Babel, J.

George, U.: vide Hellner, K. A.

Gerhard, J. P.: Nos experiences avec deux types nouveaux de lentilles cor-

néennes 274

Gloor, B. P.: Epiretinale Fibroplasie und massive praeretinale Retraktion . 227 Goder, G. und Velhagen, K.-H.: Die Bertalanffy-Methode zum Nachweis

von Tumorzellen im Blut bei Geschwülsten des Auges .... 372 Goldmann, H. und L·otmar, W.:

Retinale Sehschärfenbestimmung bei

Katarakt 175

Gonzalo-Platero, J.: vide Stangos, N.

Graupner, K.: vide Matthäus, W.

Grützner, P.: vide Ditzen, $\mathrm{K}$.

Haldimann, R. und König, H.: Ulcus corneae durch Mima polymorpha . 98

Hellner, K. A. und George, U.: über den Einfluss farbigen Lichtreizes auf

die visuell evozierten Potentiale (VEP) des optischen Cortex beim

Menschen 512

Holland, Monte G. et Cambie, E.: Une association de stigmates d'albinisme

oculaire et d'amaurose congénitale de Leber. Rapport Clinique . 425 Houber, J.-P.: Récidive

bilatérale d'une dégénérescence nodulaire de Salz-

mann après greffe perforante de la cornée 90

Huber, P. und Benz, G.: Verlaufskontrollen und Spätresultate nach opera-

tıver Behandlung praesaccaler Dakryostenosen . , . . 313 Immich, H.: vide Ditzen, K.

Jeannerod, M.: Fixation instable et nystagmus vertical 31

Kahnemann, F.: vide Rathschüler, R.

Kalberer, M.; Atar, Z. und Bider, E.: Über Augenveränderungen bei Lang-

zeitbehandlung mit Largactil 118

Keiner, E. C. J. F.: Occlusion Amblyopia 55

Kern, R. und Schärer, K.: Über eine angeborene Katarakt der Ratte. I. Lin-

senveränderungen während der ersten 14 Tage 255

Kessous-Odermatt, F. L.: vide Stucchi, C. A.

Klein, S.: vide Matthäus, W.

Klöti, R.: Erfahrungen mit der Silberklcnime bei Makulaloch-bedingten 
Netzhautablösungen 210

Koelbing, H. M.: Julius Sichel aus Frankfurt (1802-1868), ein Pionier der modernen französischen Augenheilkunde 83

Kokkinakis, K.: vide Bouzas, A.

Index VII

König, H.: vide Haldimann, R.

KozouSek, V.: ERG und Behçet-Uveitis 196

Landau, J.: vide Merin, S.

Landolt, E. und Zuccoli, A.: Mykotische Panophthalmitis nach Netzhautoperation 237

Lapis, K.: vide Radnót, M.

Lechnowitsch, Ch.: vide Comberg, D.

Leuenberg, A.: Zu $\gamma$ Photokoagulationsbehandlung der diabetischen Retino-

pathie 219

Leuenberger, P.: Modifications ultrastructurales de la rétine consécutives

à une atrophie par compression du nerf optique 157

Lotmab, W.: vide Goldmann, $\mathrm{H}$.

Madroszkiewicz, M.: Oculo-myo-dynamometry the Strength and Work of

Extraocular Muscles in Squint 491

Magora, A.: vide Zauberman, Hanan

Makabe, R.: Pilocarpintest zur Glaukomdiagnotik mit Hilfe der Tonographie 451

Makabe, R.: Über die Reaktionsdifferenz von Normal- und Glaukomaugen

auf Provokationstests 460

Martenet, Anne-Catherine: Anticorps viraux dans Гuvéite .... 243 Matthäus, W.; Klein, S.;

Graupner, K. und Dietze, H.: Vergleichende Un-

tersuchungen über die Wirksamkeit der Kälteapplikation und der

Jod-Abrasio in Kombination mit unspezifischer und virostatischer

Nachbehandlung bei der Keratitis dendritica 484

Mattheus, S.: vide Ditzen, K.

Merin, S. and Landau, J.: Abnormal Findings in Relatives of Patients with

Juvenile Hereditary Macular Degeneration (Stargardt's Disease) . 1

Nawratzki, Ilse: vide Zauberman, Hanan

Niesel, P.: Streuungen perimetrischer Untersuchungsergebnisse . . 180 Otradovec, J. and

Vladyková, J.: Zur Frage der Blutungen und der venö-

sen Stauung bei der Drusenpapille 21

Papadakis, G.: vide Bouzas, A.

Prexl, H. J.: Eine einseitige Aplasie des Nervus Opticus 11

Prijot, E.: vide Collignon, J.

Psilas, K.: vide Babel, J.

Psilas, K.: vide Ricci, A.

Psilas, K.: vide Soriano, H.

Radnót, M. and Lapis, K.: Ultrastructure of the Caruncular Oncocytoma . 63

RathschüLEr, R. et Kahnemann, F.: Modifications de vieilles méthodes

opératoires pour la correction de l'entropion et du dystichiasis . 385 Rey, P.: vide Stangos, N.

Ricci, A.; Psilas, K. et Soriano, H.: Diagnostic des tumeurs intraoculaires 
et orbitaires par échographie et par gammagraphie .... 132 Ricci, A.: vide Babel, J. Rintelen, F. und Dobrivojevic, D.: Über Gortison-Katarakt bei Nieren-

transplantation 125

Rosselet, E.; Gailloud, C. et Verrey, F.: Retinoblastome et hypopyon

(Epilogue) 139

VIII

Index

Schärer, K.: vide Kern, R.

Slezak, H.: Periphere gürtelförmige Netzhautatrophie bei Ablatio retinae . 217

Sokolió, P.: Developmental Factor in the Etiopathogenesis of Glaucomato-

cyclitic Crisis 446

Soriano, H. et Psilas, K.: Syndrome de Marchesani associé à une mégalo-

cornée et à une atrophie de Гiris; etude échographique . . . 269

Soriano, H.: vide Babel, J.

Soriano, H.: vide Ricci, A.

Stangos, N.; Rey, P. et Gonzalo-Platero, J.: Essai de standardisation d'uneméthode pour l'étude des potentiels oscillatoires chez Thomme.I. Etude morphologique 202

Stangos, N.: vide Babel. J.

Stucchi, C. A. et Bianchi, G.: Complications oculaires graves post-varicel-

leuses chez Tadulte 108

Stucchi, C. A. et Kessous-Odermatt, F. L.: Mélanose oculaire congénitale

bénigne 245

Tsacopoulos, M.: vide Babel, J.

Velhagen, K.-H.: vide Goder, G.

Verrey, F.: vide Rosselet, E.

Vladyková, J.: vide Otradovec, J.

Wieser, D.: Aspiration und Vorderkammerirrigation als Hilfe bei der Operation der Cataracta congenita 292

Witmer, R.: Therapie der Epithelinvasion der Vorderkammer nach Star-operation 286

Würth, A.: vide Breitenmoser, R.

Yunossada, M. K.: Hornhauterkrankungen bei chronischer Polyarthritis . 437

Zauberman, Hanan; Nawratzki, Ilse and Magora, A.: Surgery for Ptosis

and Strabismus in Ocular Myopathy 477

Zuccoli, A.: vide Landolt, E.

Proceedings - Verhandlungsberichte - Congrès

Schweizerische Ophthalmologische Gesellschaft, 62. Generalver-sammlung vom 11. bis 13.

September 1969 in Biel - Société Suissed'Ophtalmologie, 62e Assemblée générale du 11 au 13

septembre1969 à Bienne 81

Obituary - Nachruf - Necrologue

Paul Bailliart 413

Book Reviews - Buchbesprechungen - Livres nouveaux . 78,356,413

Letter to the Editors 418

Varia 360,422

I. Index rerum ad Vol. $161 \quad 534$

Index autorum ad Vol. 161543 